EPJ Web of Conferences 16, 07003 (2011)

DOI: $10.1051 /$ epjconf/20111607003

(C) Owned by the authors, published by EDP Sciences, 2011

\title{
Imaging extrasolar planets with the European Extremely Large Telescope
}

\author{
S. Gladysz ${ }^{1}$,, B.F. Castella ${ }^{2}$, R. Rebolo ${ }^{2}$, M. Kissler-Patig ${ }^{1}$ and L. Jolissaint ${ }^{3}$ \\ ${ }^{1}$ European Southern Observatory, Karl-Schwarzschild-Strasse 2, 85748 Garching, Germany \\ 2 Instituto de Astrofisica de Canarias, C/ Via Lactea, s/n, 38205 La Laguna, Spain \\ ${ }^{3}$ Leiden Observatory, University of Leiden, Niels Bohrweg 2, 2333 CA Leiden, \\ The Netherlands
}

\begin{abstract}
The European Extremely Large Telescope (E-ELT) is the most ambitious of the ELTs being planned. With a diameter of $42 \mathrm{~m}$ and being fully adaptive from the start, the E-ELT will be more than one hundred times more sensitive than the present-day largest optical telescopes. Discovering and characterising planets around other stars will be one of the most important aspects of the E-ELT science programme. We model an extreme adaptive optics instrument on the E-ELT. The resulting contrast curves translate to the detectability of exoplanets.
\end{abstract}

\section{INTRODUCTION}

Out of the three optical Extremely Large Telescope (ELT) projects [1-3] the E-ELT is the most ambitious. The present concept features as a baseline a $42 \mathrm{~m}$ primary mirror composed of 984 segments, each $1.45 \mathrm{~m}$ wide. The adaptive optics (AO) system built into the telescope is composed of two mirrors: a deformable M4 mirror, $2.5 \mathrm{~m}$ in diameter and supported by 5000 or more actuators, and a tip-tilt mirror, $\mathrm{M} 5,2.7 \mathrm{~m}$ in diameter.

Ten instrument studies are underway, including two post-focal AO modules for wide-field turbulence compensation. Out of these instrument concepts two or three are likely to be ready for "first light" which, with decision to build expected in 2010, would happen in 2018.

Direct imaging and spectroscopy of exoplanets requires, for the most challenging and interesting objects, eXtreme Adaptive Optics (XAO). The XAO instrument for detection and characterization of exoplanets on the E-ELT is EPICS [4]. The study of exoplanets motivates the design of HARMONI [5] and METIS [6] too, although these two instruments will rely on modest AO correction provided only by the telescope (METIS will additionally rely on long wavelengths to deliver high Strehl ratio).

In this paper we do not focus on any specific instrument design. The goal of this work is to sample a large parameter space, and not to have a full end-to-end model of one planned E-ELT instrument. We want to know how parameters such as: the level of AO correction, efficiency of coronagraphic diffraction suppression, and distance to the target impact the final contrast curves. The contrast curves tell us which exoplanets are detectable at a given separation from a parent star.

\section{DESCRIPTION OF THE SIMULATIONS}

All simulations are polychromatic, and they assume the same spectral range: $0.9-1.7 \mu \mathrm{m}$. This range was chosen because it contains the most interesting molecular bands: $\mathrm{O}_{2}, \mathrm{CO}_{2}, \mathrm{H}_{2} \mathrm{O}$, and $\mathrm{CH}_{4}$. We achieve

\footnotetext{
ae-mail: sgladysz@eso.org
}

This is an Open Access article distributed under the terms of the Creative Commons Attribution-Noncommercial License 3.0, which permits unrestricted use, distribution, and reproduction in any noncommercial medium, provided the original work is properly cited. 


\section{EPJ Web of Conferences}

high dynamic range in the final, single image through three simulated techniques:

- Image sharpening through turbulence compensation with XAO (0.2 m inter-actuator spacing),

- Diffraction suppression through coronagraphy. It should be mentioned here that efficient coronagraphic starlight suppression is helped by first reducing the image to a narrow spot with XAO, i.e. the second step in our simulations is intrinsically linked to the first. If XAO works as expected the diffraction-limited spot hits the focal-plane mask and most of the light is blocked.

- Finally we implement a post-processing technique to remove the residual stellar light from the spectral data cube. Here we use the method called spectral deconvolution [7] which has been used on spectral data cubes recorded with Integral Field Spectrographs (IFS). In all our simulations we assume an IFS is present but we do not have a detailed model of light propagation through a spectrograph. Such a model depends on the design of an IFS (BIGRE or TIGER configuration, see [8]) and is certainly outside the scope of this work. For the same reason we do not model Fresnel propagation, which depends on the number and relative separations of optical surfaces within an instrument.

For the wavefront sensor (WFS) we implemented the Shack-Hartmann device. The action of AO is modelled in an analytic fashion. For example the AO fitting error can be described as a result of the high-pass transfer function acting on the Kolmogorov atmospheric power spectrum of phase. Stars of various magnitudes (bolometric 6 to 10, covering spectral types $\mathrm{G}, \mathrm{K}$ and $\mathrm{M}$, or distances 10 to $50 \mathrm{pc}$ ) are assumed to be observed on-axis therefore we consider the transfer functions corresponding to the fitting error, the WFS noise error, and the AO loop delay error, but neglect the anisoplanatic transfer function. The WFS aliasing effect is also neglected because we assume a spatial filter in front of the WFS. The PAOLA simulation package [9] was used to model the action of AO on the atmospheric power spectrum. The modelled atmosphere consists of 10 layers. The heights, fractional strengths, and wind speeds in the layers were taken from the model which is being extensively used in simulations of the E-ELT, as part of the E-ELT Design Reference Mission [10]. Seeing was set to $0.7 \operatorname{arcsec}$ at $500 \mathrm{~nm}$. Scintillation was neglected.

The AO-filtered phase spectrum was used to generate independent realizations of AO residual phase using a classic, Fourier-domain filtering algorithm. To this phase two static components have been added: static aberrations from the main mirror's segments (originally $50 \mathrm{~nm}$ total rms divided evenly between the piston, tip/tilt, coma, and astigmatism contributions; subsequently filtered by AO to give $15 \mathrm{~nm}$ rms of residual mirror aberrations reaching the coronagraph), and the non-common-path aberrations not sensed by AO and modelled with an $\mathrm{f}^{-2}$ spectrum [11] and the rms value of $30 \mathrm{~nm}$. The instantaneous PSFs corresponding to these wavefronts had Strehl ratios between 80 and $95 \%$, for the spectral range $0.9-1.7 \mu \mathrm{m}$.

To simulate an IFS we needed to have the wavelength-scaled wavefronts. After one AO-corrected wavefront is generated for the first wavelength of $0.9 \mu \mathrm{m}$, the programme loops over the 100 channels in the spectral range, scaling the stored phase by each channel's wavelength:

$$
\phi\left(\lambda_{i}\right)=\phi\left(\lambda_{0}\right) \cdot \lambda_{0} / \lambda_{i}
$$

where $\phi$ stands for phase of the wavefront, and $\lambda$ is wavelength $\left(\lambda_{0}=0.9 \mu \mathrm{m}\right)$. In the simulations this loop over wavelengths is contained inside the loop over time: 500 iterations over instantaneous wavefronts; assuming an atmospheric coherence time of $20 \mathrm{~ms}$ within our spectral range this gives $10 \mathrm{sec}$ of observing time. Equation 1 excludes differential chromatic aberrations between the channels. To keep the constant plate scale $(\lambda / 2 D$ at $0.9 \mu \mathrm{m})$ the wavefronts were embedded in ever-increasing arrays of zeros before Fourier-transforming.

The action of a Lyot-style coronagraph is simulated in the following way. For each wavelength the electric field is Fourier-transformed to give the amplitude PSF in the focal plane. The result is multiplied by the representation of the focal-plane mask, taken to be $7.5 \lambda / D$ in diameter. This value has been 


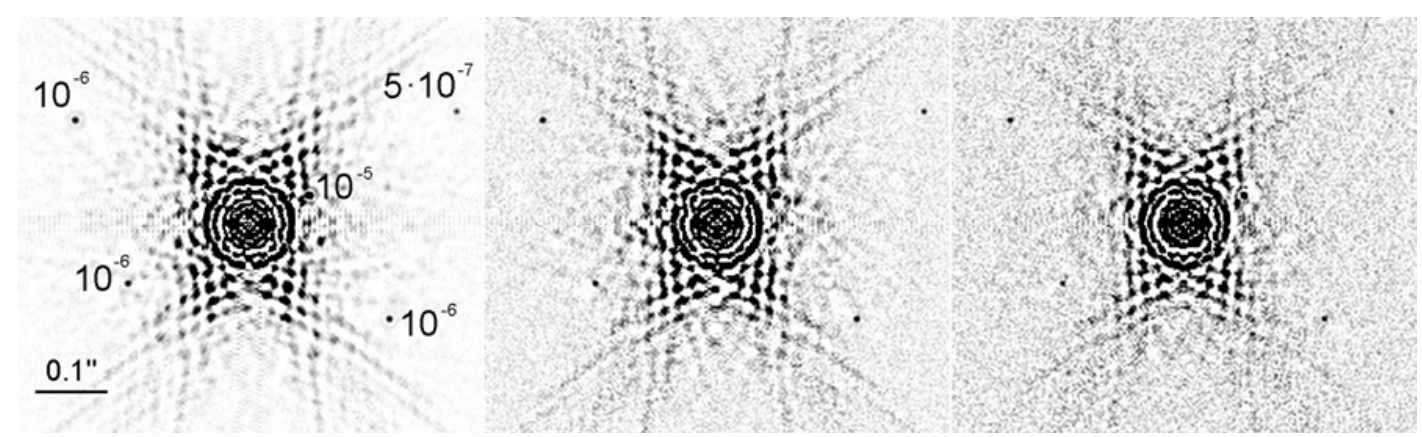

Figure 1. Final 10 sec-long images after spectral deconvolution, for G2-type stars at distances 10 (left), 30 (centre) and $50 \mathrm{pc}$ (right panel). This zoomed-in part of the field-of-view contains the closest five of the seven test planets. Images are displayed on the linear scale; the central parts are deliberately saturated to bring out the faint speckle structure outside the PSF core. Colours are inverted for better contrast.

optimized for the inner working angle of $3.9 \lambda / D$ and the current design of the E-ELT main mirror, i.e. $30 \%$ linear obscuration ratio [12]. The electric field after the focal-plane mask is inverse Fouriertransformed and multiplied by the model of the Lyot stop. For the Lyot coronagraph this stop has an oversized central obscuration and undersized outer edge, resulting in the throughput of $67 \%$. Fourier transform of the field after the Lyot stop gives the electric field in the detector plane, and the squared modulus of this Fourier transform yields the final on-axis instantaneous PSF. For the images of test planets the electric field corresponding to AO-residuals and instrumental aberrations is propagated only through the Lyot stop, giving the off-axis PSF. We scale and shift this off-axis PSF to simulate seven planets located at various separations from the star, within the range of $0.1-0.5 \mathrm{arcsec}$. The contrast ratios are between $10^{5}$ and $10^{7}$. These instantaneous images of planetary systems are stored for each channel and then co-added, resulting in a spectral data cube with 100 images, each $10 \mathrm{sec}$ long. Finally, we loop over this cube adding Poisson, background $\left(15 \mathrm{mag} / \mathrm{arcsec}^{2}\right)$ and readout noise $\left(10 \mathrm{e}^{-}\right)$.

Spectral deconvolution is applied to this data cube to increase the contrast by approximately two orders of magnitude and recover the signal of the faintest test planets. Briefly: in the simulated data cube the residuals of the Airy pattern expand with wavelength, and the planet stays fixed. Each image in the cube is spatially scaled by Fourier interpolation to fit a common grid, so that the Airy rings are now aligned and the planet's position changes through the cube: it gets closer to the star with increasing wavelength. A 2nd-order polynomial is fitted for each spaxel (spectral vector in the scaled cube). This particular choice of the polynomial order is justified by the following argument: The wavefront expressed in units of length, which to a first order can be assumed to have the same optical path difference across the 100 channels, is scaled by $2 \pi / \lambda$ to give phase. Theoretically, response of intensity for one constant location on an Airy ring to change in wavelength of the wavefront should be $\sim(2 \pi / \lambda)^{2}$, because image intensity is the power spectrum of the electric field. This argument ignores changes in PSF morphology which naturally occur for a wide wavelength range where the Strehl ratio changes significantly. Nevertheless these changes are smooth when one considers many channels, as we do here, and can be fitted with a moderately smooth polynomial. After the first fitting step, any outliers (possible planets) are removed from the vector, and this procedure is repeated until no value in the sample exceeds the fit by two standard errors. Once the fit is not skewed anymore by possible signals, it is subtracted from the spaxel under consideration - this step removes most of the stellar modulation, but leaves possible planetary signal intact. The images are then re-scaled to the original grid, so that now a planet would be aligned on the same pixel again. Finally the cube is collapsed to produce one final image. This image (see Figure 1) is processed to yield azimuthally averaged contrast curves - giving $5 \sigma$ detection limits as a function of angular separation from a star. 
EPJ Web of Conferences

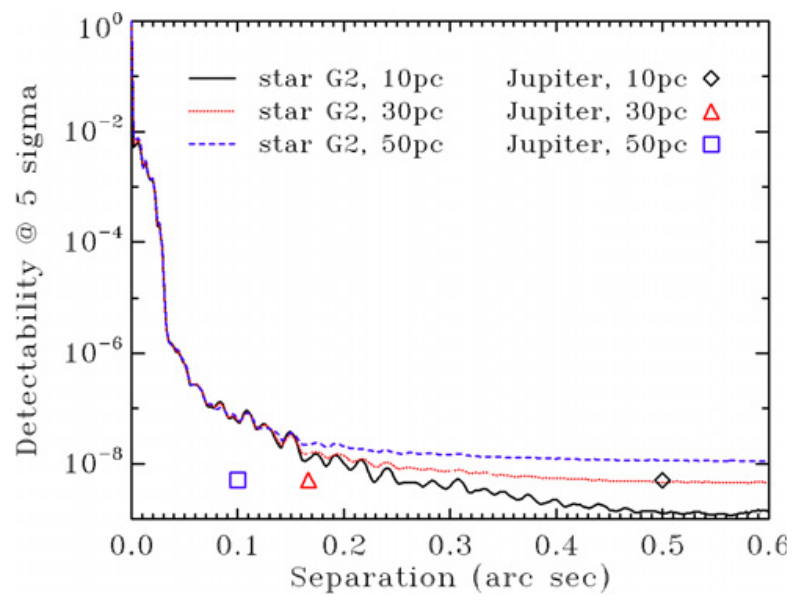

Figure 2. Contrast curves scaled from 10 sec to 10 hours. For comparison contrast for an "old \& cold", Jupiter-like planet (mass $1 \mathrm{M}_{\mathrm{J}}$, orbital radius 5AU) is over-plotted.

\section{RESULTS}

We show here some results, noting that these are only examples of a wide spectrum of results we have obtained by changing, for example, the values of seeing, stellar magnitude, coronagraph type or noise levels.

Figure 1 shows the outputs of three simulations where we tested the influence of distance to the star or equivalently apparent stellar brightness. Contrast, shown in Figure 2, degrades with distance to the target. Close to the optical axis contrast is limited by non-linear spatial scaling of the diffraction pattern with wavelength, induced by the Lyot coronagraph. This can be alleviated by using a less chromatic coronagraph [12]. Further away from the core we believe $10^{9}$ is the limit, i.e. highest achievable contrast to be obtained with this particular XAO configuration for the E-ELT. This means that obtaining images of Earth-like planets (contrast of around $10^{10}$ ), even during times when seeing is significantly better than 0.7 arcsec considered here, would be extremely serendipitous.

\section{References}

[1] http://www.eso.org/sci/facilities/eelt

[2] http://www.tmt.org

[3] http://www.gmto.org

[4] Kasper, M. et al., SPIE Proceedings 7015, (2008) 70151S

[5] Tecza, M., Thatte, N., Clarke, F., Freeman, D., Science with the VLT in the ELT Era, (2009) 267

[6] Brandl, B. et al., SPIE Proceedings 7014, (2008) 70141N

[7] Sparks, W., Ford, H., Astrophysical Journal 578, (2002) 543

[8] Antichi, J. et al., Astrophysical Journal 695 (2009) 1042

[9] Jolissaint, L., Veran, J.-P., Conan, R., Journal of the Optical Society of America A 23, (2006) 382

[10] http://www.eso.org/sci/facilities/eelt/science/drm/tech_data/ao

[11] Cavarroc, C., Boccaletti, A., Baudoz, P., Fusco, T., Rouan, D., Astronomy and Astrophysics 447, (2006) 397

[12] Martinez, P., Boccaletti, A., Kasper, M., Cavarroc, C., Yaitskova, N., Fusco, T., Verinaud, C., Astronomy and Astrophysics 492, (2008) 289 